\title{
Genomic and experimental evidence suggests that Verrucomicrobium spinosum interacts with eukaryotes
}

\author{
Michelle Sait ${ }^{1+}$, Olga K. Kamneva ${ }^{1}$, David S. Fay ${ }^{1}$, Natalia V. Kirienko ${ }^{1+}$, James Polek ${ }^{1}$, \\ Mimi M. Shirasu-Hiza ${ }^{2+}$ and Naomi L. Ward ${ }^{1 *}$
}

1 Department of Molecular Biology, University of Wyoming, Laramie, WY, USA

2 Department of Microbiology and Immunology, Stanford University, Stanford, CA, USA

\author{
Edited by: \\ Frank T Robb, University of California, \\ USA

\section{Reviewed by:} \\ Garry Myers, University of Maryland, \\ USA \\ Nils-Kaare Birkeland, University of \\ Bergen, Norway \\ *Correspondence: \\ Naomi L. Ward, Department of \\ Molecular Biology, University of \\ Wyoming, Dept 3944, 1000 E. \\ University Avenue, Laramie, WY \\ 82071, USA. \\ e-mail: n/ward@uwyo.edu

\section{${ }^{+}$Present address:} \\ Michelle Sait, Moredun Research \\ Institute, Pentlands Science Park, \\ Bush Loan, Edinburgh, Midlothian, \\ UK; \\ Natalia V. Kirienko, Department of \\ Molecular Biology, Massachusetts \\ General Hospital, Boston, MA, USA; \\ Department of Genetics, Harvard \\ Medical School, Boston, MA, USA; \\ Mimi M. Shirasu-Hiza, Department of \\ Genetics and Development, Columbia \\ University Medical Center, New York, \\ NY, USA.
}

Our knowledge of pathogens and symbionts is heavily biased toward phyla containing species that are straightforward to isolate in pure culture. Novel bacterial phyla are often represented by a handful of strains, and the number of species interacting with eukaryotes is likely underestimated. Identification of predicted pathogenesis and symbiosis determinants such as the Type III Secretion System (T3SS) in the genomes of "free-living" bacteria suggests that these microbes participate in uncharacterized interactions with eukaryotes. Our study aimed to test this hypothesis on Verrucomicrobium spinosum (phylum Verrucomicrobia) and to begin characterization of its predicted T3SS. We showed the putative T3SS structural genes to be transcriptionally active, and that expression of predicted effector proteins was toxic to yeast in an established functional screen. Our results suggest that the predicted T3SS genes of $V$. spinosum could encode a functional T3SS, although further work is needed to determine whether $V$. spinosum produces a T3SS injectisome that delivers the predicted effectors. In the absence of a known eukaryotic host, we made use of invertebrate infection models. The injection or feeding of $V$. spinosum to Drosophila melanogaster and Caenorhabditis elegans, respectively, was shown to result in increased mortality rates relative to controls, a phenomenon exaggerated in $C$. elegans mutants hypersensitive to pathogen infection. This finding, although not conclusively demonstrating pathogenesis, suggests that $V$. spinosum is capable of pathogenic activity toward an invertebrate host. Symbiotic interactions with a natural host provide an alternative explanation for the results seen in the invertebrate models. Further work is needed to determine whether $V$. spinosum can establish and maintain interactions with eukaryotic species found in its natural habitat, and whether the predicted T3SS is directly involved in pathogenic or symbiotic activity.

\section{Keywords: Verrucomicrobia, genome, eukaryotic host}

\section{INTRODUCTION}

For anthropocentric reasons, bacteria are often conceptually divided into free-living and host-associated organisms. However, we know that different members of the same bacterial phylum can exhibit a multitude of lifestyles (free-living, pathogen, symbiont), and even that a single species can engage in different kinds of relationships with different hosts. It is also clear that bacterial phyla containing known pathogens and symbionts are enormously over-represented in our culture collections and sequence databases (Martiny and Field, 2005), whereas our knowledge of many novel phyla is limited to a handful of strains. The relatively obscure bacterium Verrucomicrobium spinosum (phylum Verrucomicrobia) was isolated from a lake in northern Germany (Schlesner, 1987), and its most interesting feature until now has been its unusual cellular morphology, featuring wart-like cellular protrusions (Schlesner, 1987) and a compartmentalized cell plan shared with the planctomycetes (Lee et al., 2009). For two decades, $V$. spinosum has been regarded as a free-living, non-pathogenic microbe, but its genome sequence contains a predicted Type III secretion system (T3SS; Pallen et al., 2005). T3SS is a hallmark of bacteria-eukaryote interactions, chiefly known for its role as a pathogenesis factor, delivering toxic effector proteins via direct cell-to-cell contact with eukaryotic cells, but also involved in symbioses (Galán and Wolf-Watz, 2006). It has been suggested that "free-living" organisms possessing predicted T3SS may participate in uncharacterized interactions with eukaryotes (Pallen et al., 2005). Our study aimed to test this hypothesis on Verrucomicrobium spinosum and to begin characterization of its predicted T3SS. We achieved our aims by generating experimental data on the transcriptional activity and protein-protein interactions of T3SS genes and their gene products, respectively. We also demonstrated that $V$. spinosum can kill two different model invertebrate hosts.

\section{RESULTS \\ V. SPINOSUM POSSESSES AND EXPRESSES PREDICTED TYPE III SECRETION SYSTEM GENES}

The V. spinosum genome contains all required structural components for a functional T3SS (Figure 1; Table A1 in Appendix). The V. spinosum T3SS genes are found in a cluster with strikingly similar organization to other T3SS gene clusters, particularly the 


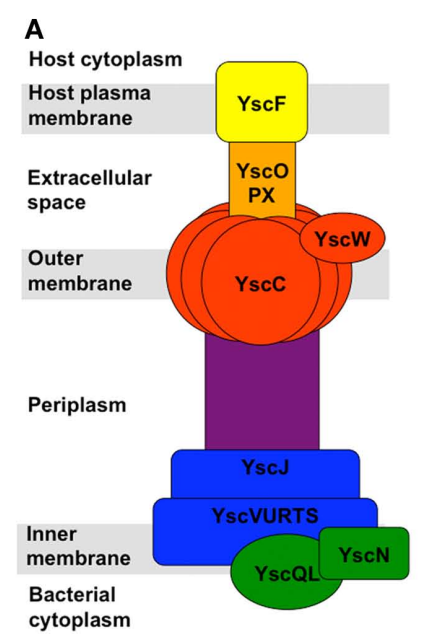

FIGURE 1 | Verrucomicrobium spinosum possesses genes predicted to encode a T3SS. (A) General organization of T3SS structural components in experimentally characterized T3SS, labeled according to the Yersinia T3SS nomenclature. (B) Organization of predicted T3SS structural genes in the genomes of $V$. spinosum, Desulfovibrio vulgaris, Lawsonia intracellularis, and Chlamydophila

\section{B}
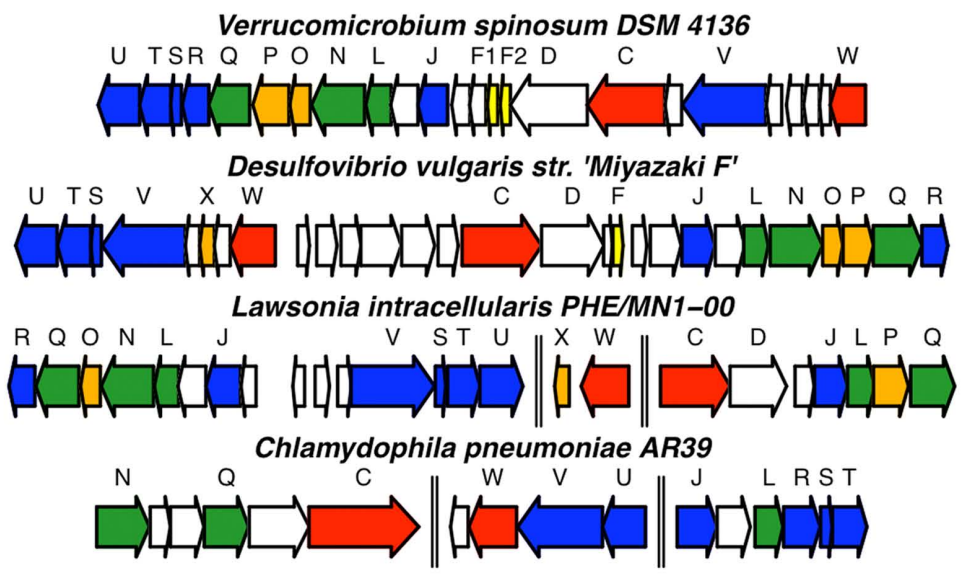

well-characterized T3SS found in Yersinia species, and the T3SS of desulfovibrios and related organisms (Heidelberg et al., 2004; Pallen et al., 2005). All essential categories of T3SS structural proteins were identified, including membrane anchors (VspC, VspQ), candidate needle proteins (VspF1, VspF2), needle protein chaperones (VspE, VspG), and an ATPase (VspN; Table A1 in Appendix). Importantly, we found that several of these genes were actively transcribed. Transcription of predicted outer and inner bacterial membrane anchors of the T3SS, and candidate needle proteins, was detected by RT-PCR (Figure 2) under standard growth conditions (described in Materials and Methods). Furthermore, specialized cytoplasmic chaperones are usually required for the stability of the T3SS, and the efficient translocation of T3SS components and effectors (Galán and Wolf-Watz, 2006). We demonstrated a physical association between one needle protein (VspF1) and one chaperone (VspE; Table A2 in Appendix) by yeast two-hybrid screening, thus supporting a functional interaction.

\section{EXPRESSION OF PREDICTED V. SPINOSUM T3SS EFFECTOR PROTEINS SUPPRESSES GROWTH OF YEAST}

We next used a yeast functional screen (Lesser and Miller, 2001) to experimentally confirm the toxicity of predicted T3SS effector proteins from $V$. spinosum. Expression of T3SS effectors, but not other bacterial proteins, causes growth inhibition of Saccharomyces cerevisiae (Lesser and Miller, 2001; Slagowski et al., 2008). We identified three candidate effectors, based on their proximity to the T3SS structural gene cluster, sequence similarity to known T3SS effectors, and/or sequence properties reported to be characteristic of T3SS effectors (Schechter et al., 2006). Two of these, open reading frame $(\mathrm{ORF}) 05930\left(p=7.9\right.$ e $10^{-17} ; t$-test $)$ and ORF04374 ( $p=3.3$ e $10^{-7} ; t$-test), showed a significant inhibition of growth under inducing conditions (Figure 3). Expression of control V. spinosum genes showed no such effect. While we have pneumoniae. The latter is included because the chlamydiae are generally considered to be more phylogenetically related to the Verrucomicrobia than other phyla. Genes are colored to correspond to the protein components depicted in part $(\mathbf{A})$ of the figure. Double vertical lines indicate that the T3SS genes are not immediately adjacent to each other in the respective genomes.

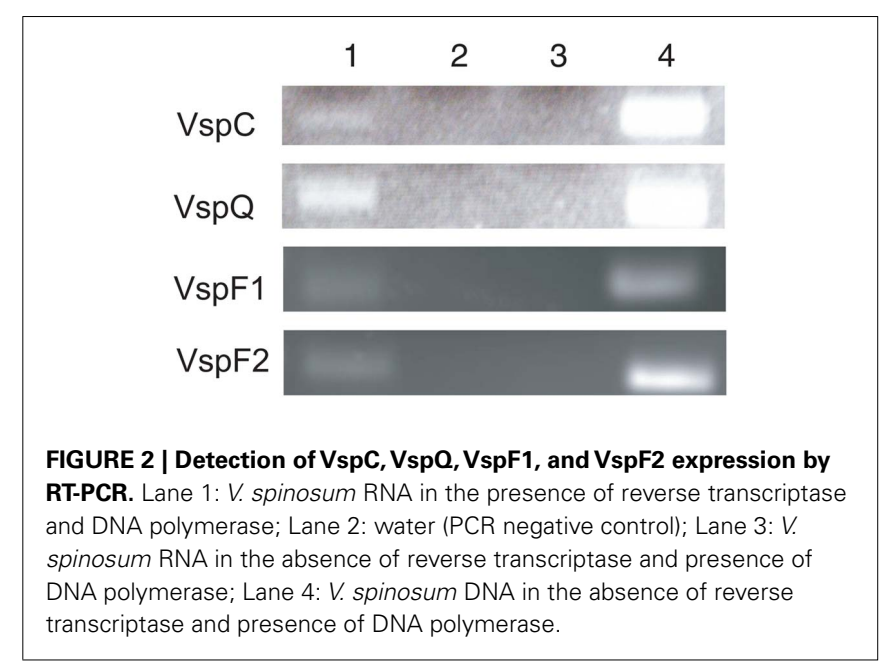

not directly demonstrated that the predicted T3SS secretes these predicted effectors, our data suggest that injection of these effectors into eukaryotic cells would be toxic, similar to other known T3SS effectors.

\section{SPINOSUM INCREASES MORTALITY OF D. MELANOGASTER}

We next performed standard infection assays (Schneider et al., 2007) with V. spinosum in Drosophila melanogaster. Drosophila is an established model organism for examining T3SS-mediated pathogenesis (Brandt et al., 2004). Although non-pathogenic bacteria can elicit an immune response when injected into $D$. melanogaster (Lemaitre et al., 1997), they have no effect on fly mortality (Schneider et al., 2007). Wild-type (Oregon R) flies infected with $V$. spinosum died more quickly than flies injected with buffer alone (Figure 4), with approximately 40\% survival after 20 days, 


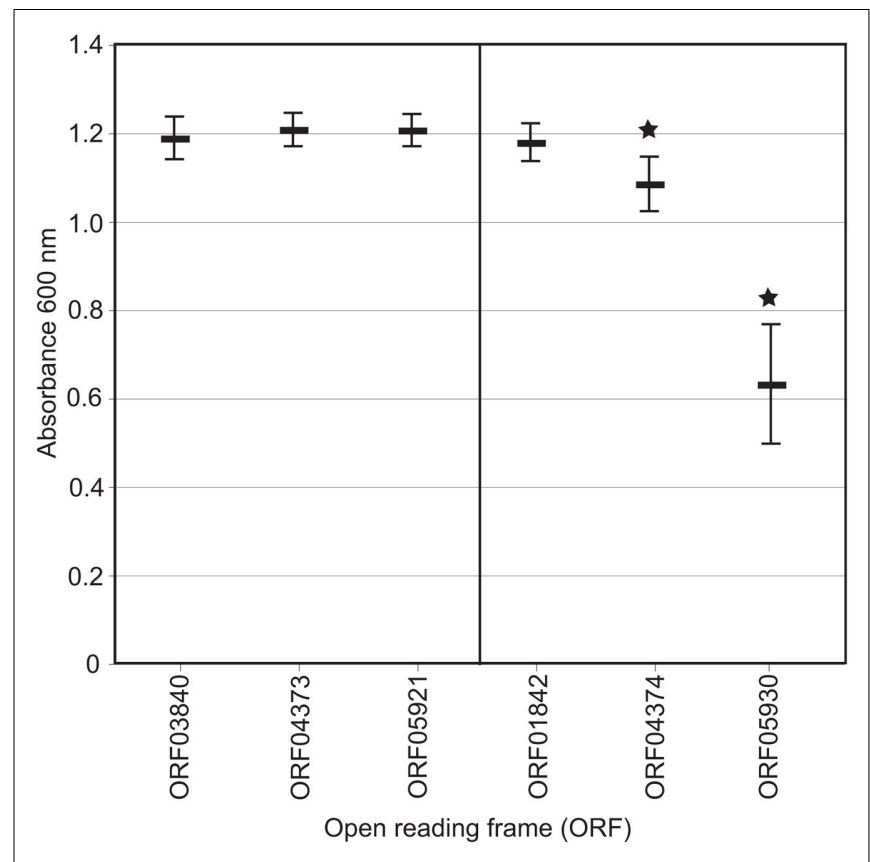

FIGURE 3 | Optical density of yeast cultures expressing putative effector genes (on right) and negative control genes (on left). Vertical bar represents distribution of values for 12 individual clones repeated in duplicate. ${ }^{*} p=7.9 \mathrm{e}^{-17}$ (ORF05930, $t$-test) and 3.3 e10 ${ }^{-7}$ (ORF04374, $t$-test).

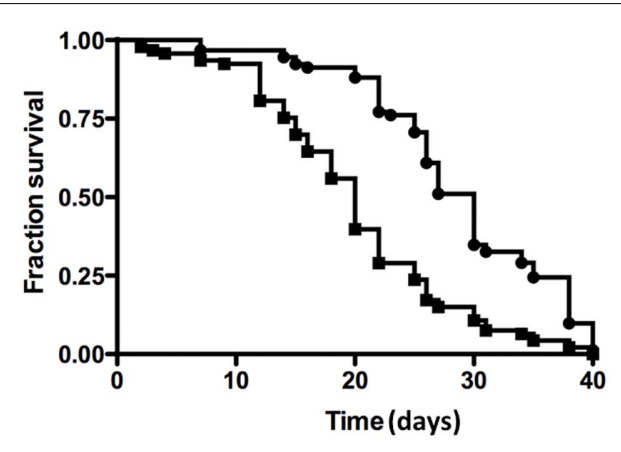

FIGURE 4 | Drosophila melanogaster survival assays for $\boldsymbol{V}$. spinosum (squares) and PBS negative control (diamonds). Each experiment comprised 100 male wild-type Oregon $\mathrm{R}$ flies. Infected flies died faster than control flies according to log-rank analysis $(p<0.0001)$.

compared to $85 \%$ survival for negative control buffer-injected flies $\left(p=<1.0 \times 10^{-4}\right)$. A similar effect observed using wild-type Salmonella typhimurium was shown to be dependent on the T3SS (Brandt et al., 2004).

\section{SPINOSUM INCREASES MORTALITY OF C. ELEGANS}

We also examined the effect of $V$. spinosum exposure on the mortality of Caenorhabditis elegans. Sterile mutant worms [fer-15(b26); fem-1(hc17)] exposed to living $V$. spinosum exhibited increased mortality relative to control worms feeding on Escherichia coli (Figure 5A), whereas heat-killed $V$. spinosum cells had no such effect (Figure A1A in Appendix). This suggested a direct and adverse interaction between living $V$. spinosum and the worm rather than a non-specific mortality increase due to provision of a nutritionally inferior diet or similar non-specific effect. $V$. spinosum-induced mortality was increased (Figure 5B) in a worm deletion mutant [ $f$ shr-1(ok778)] previously shown to be hypersensitive to pathogen infection by multiple agents (Powell et al., 2009). Using a multi-copy extrachromosomal array that overexpresses fshr-1 (Cho et al., 2007), we were able to completely reverse $V$. spinosum-associated death in fshr-1(ok778) mutants (Figure 5B). In fact, overexpression of $f_{s h} r-1$ substantially reduced mortality

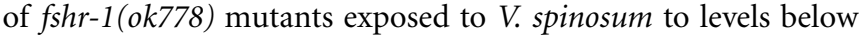
that observed for wild-type, similar to our observations with the known pathogens, S. aureus, and E. faecalis (Figures A1B,C in Appendix). In contrast, $f$ sh $r$ - 1 expression levels do not correlate to life expectancy on a non-pathogenic strain (Figure A1D in Appendix), indicating that the effects of $f$ shr- 1 on survival are specific to pathogen exposure (Powell et al., 2009).

CAN WE PREDICT THE NATURAL EUKARYOTIC HOST OF V. SPINOSUM? Phylogenetic analysis of structural T3SS proteins has shown most bacteria possessing T3SS to cluster according to their specific interaction type, such as plant pathogen, obligate intracellular animal pathogen, or extracellular animal pathogen (Troisfontaines and Cornelis, 2005). In an attempt to predict the nature of any uncharacterized $V$. spinosum-eukaryote interaction, we conducted phylogenetic analysis of YscN-like sequences (the T3SS ATPase from Yersinia) from V. spinosum, all available free-living bacteria carrying predicted T3SS genes, and reference pathogens and symbionts (Figure 6). The V. spinosum $\mathrm{YscN}$ appeared most closely related to those from strains of three deltaproteobacteria: Lawsonia intracellularis, Desulfovibrio piger and Desulfovibrio vulgaris. This cluster was recovered with analysis of some, but not all, additional T3SS genes (data not shown). L. intracellularis is an obligate intracellular pathogen and causes proliferative enteritis in pigs (Kroll et al., 2005). Members of the genus Desulfovibrio are anaerobic sulfate-reducing bacteria and include both commensals and pathogens of the human gastrointestinal tract (Gibson et al., 1990, 1991; Willis et al., 1997; Zinkevich and Beech, 2000; Loubinoux et al., 2002; Goldstein et al., 2003), as well as free-living environmental bacteria. Specifically, strains of D. piger and D. vulgaris are human intestinal commensals and $D$. piger also acts as an occasional human opportunistic pathogen. Genes predicted to encode T3SS in the L. intracellularis and D. vulgaris genomes have been described previously (Heidelberg et al., 2004; Pallen et al., 2005), and the T3SS of L. intracellularis has recently been linked to pathogenesis in the porcine host (Alberdi et al., 2009). There are no reports of infection of invertebrate models with these organisms, so it is not clear whether their host range could extend to invertebrates.

\section{DISCUSSION}

We have begun to address two questions, using Verrucomicrobium spinosum as a test case. The first question is whether predicted T3SS genes without established function in "free-living" bacteria have the potential to encode a functional T3SS, i.e., an injectisome that translocates effector proteins into a eukaryotic host cell. Our results showed that the predicted T3SS genes of V. spinosum 


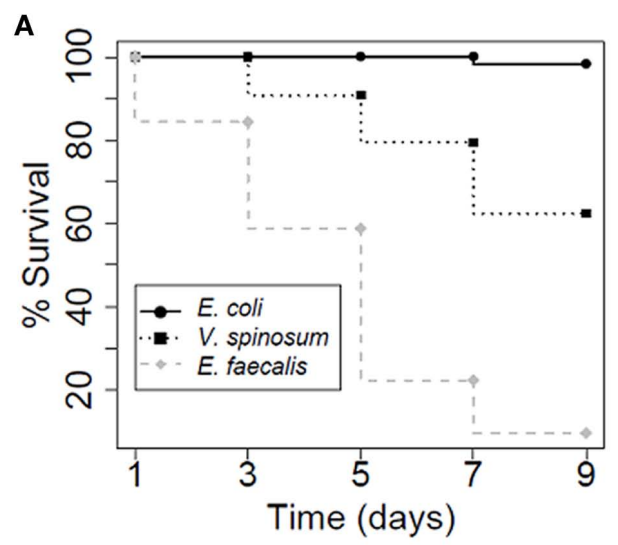

FIGURE 5 | Caenorhabditis elegans survival assays. Each experiment comprised of approximately 100 worms. (A) CF512 worms (sterile mutants) exposed to $E$. faecalis and $V$. spinosum died faster than CF512 worms exposed to $E$. coli, according to log-rank analysis ( $p=0$ for $E$. faecalis and

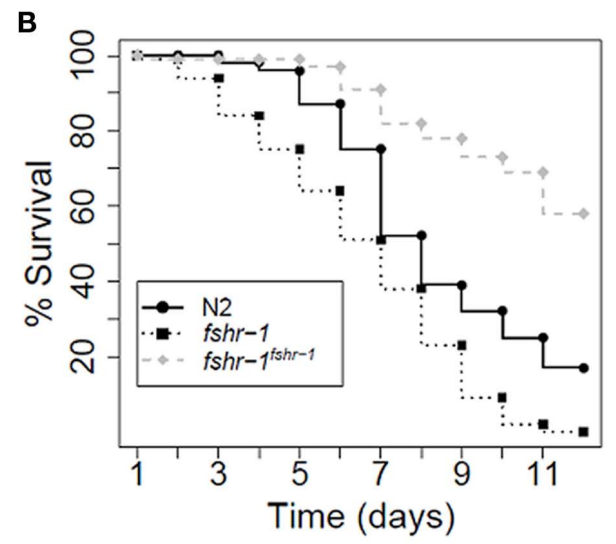

$2.33 e^{-15}$ for $V$. spinosum). (B) When exposed to $V$. spinosum, worm mutants hypersensitive to pathogens $(f s h r-1)$ died faster than $\mathrm{N} 2$ wild-type worms $\left(p=5.04 \mathrm{e}^{-7}\right)$. Worms over-expressing an $f s h r-1$ multi-copy array died more slowly than N2 wild-type worms $\left(p=7.18 \mathrm{e}^{-13}\right)$.

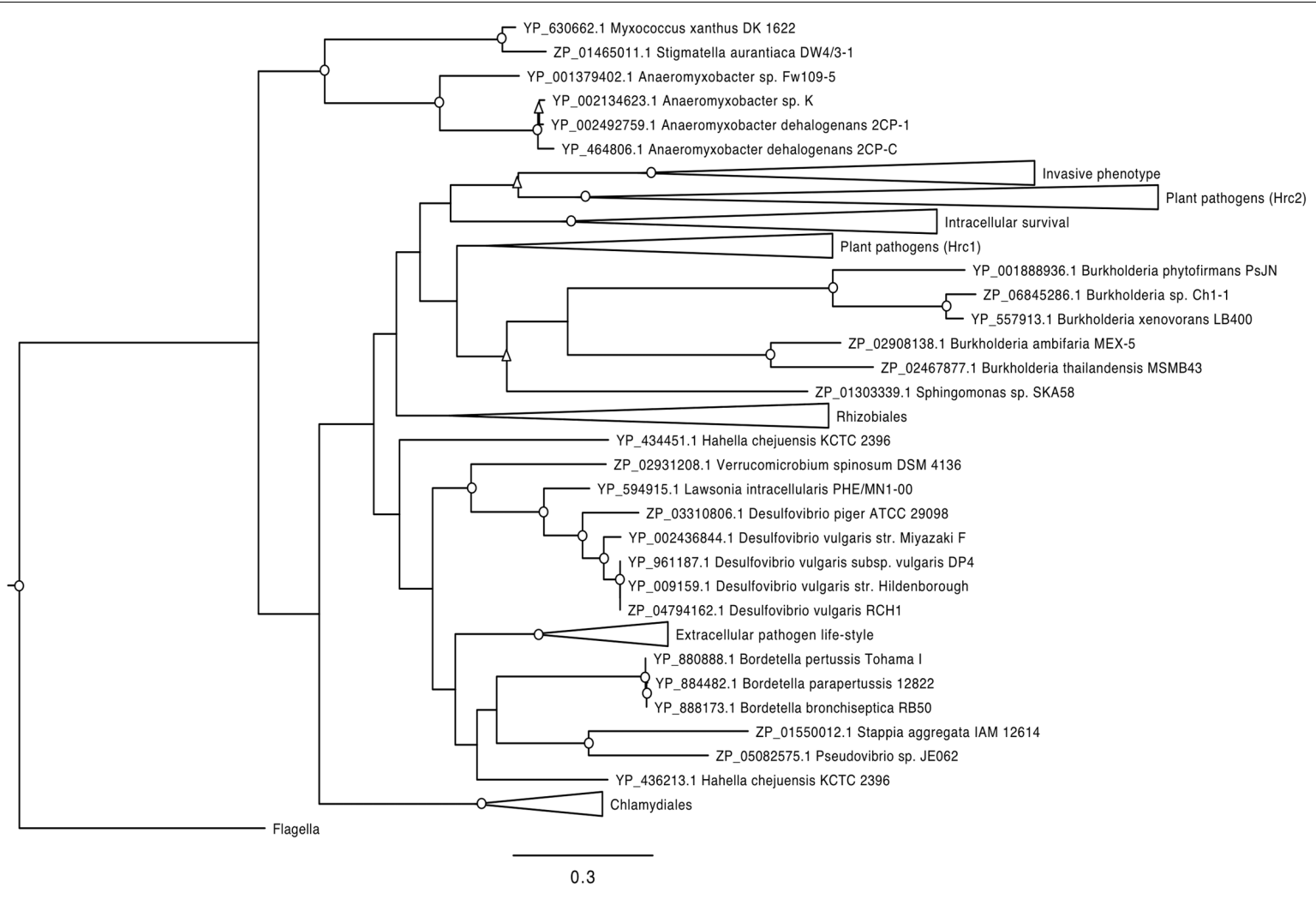

FIGURE 6 | Phylogenetic relationship of T3SS ATPases from V. spinosum and representatives of other bacterial groups containing T3SS; the ATPase group associated with the flagellar apparatus was used as an outgroup. Bootstrap values obtained based on 1000 bootstrap runs are indicated by: $O$ above $80 \%, \Delta$ between 80 and $60 \%$; values less than $60 \%$ are not shown. Bar denotes 0.3 amino acid substitutions per site. Collapsed clades indicate T3SS classes as described by Troisfontaines and Cornelis (2005). Hrc1 and Hrc2 plant pathogen groups; intracellular survival; invasive phenotype; Chlamydiales T3SS family (intracellular life-style); extracellular pathogen life-style, resistance to phagocytosis, and triggering of apoptosis in macrophages; Rhizobiales T3SS family (symbiotic relations with leguminous plants). Open clusters contain at least one "free-living" organism. are transcriptionally active and identify a candidate chaperone for one of two predicted T3SS needle proteins. We also demonstrated that expression of $V$. spinosum proteins inhibits growth in an established yeast functional screen for T3SS effectors. Although 
the growth inhibition appears modest (Figure 3), use of this screen with characterized virulence proteins from a known pathogen (Shigella flexneri) showed that growth inhibition was variable (up to $50 \%$ compared to controls; Slagowski et al., 2008). Thus the $50 \%$ growth inhibition observed for V. spinosum ORF05930 corresponded to the most toxic protein screen result from $S$. flexneri. These findings represent only first steps toward characterizing the putative V. spinosum T3SS. Future work includes determining whether T3SS genes are expressed at the protein level, whether the resulting proteins assemble to form an injectisome, and whether the injectisome translocates the predicted effectors into a host cell. It will also be necessary to pursue approaches complementary to yeast two-hybrid screens to characterize chaperone interactions, as it is possible that the very nature of chaperones leads them to interact non-specifically with poorly folded proteins.

The second question is whether $V$. spinosum can interact with eukaryotes. The results of our infection studies in two different model invertebrates suggest that it can. In particular, the increased mortality of C. elegans $f_{s h}$ - 1 deletion mutants exposed to $V$. spinosum, relative to wild-type worms, strongly argues for a pathogenic interaction in this model. However, we cannot presently extrapolate this result to the natural host(s) of $V$. spinosum, given that mechanisms of bacterial pathogenesis (e.g., T3SS) are also used to initiate and maintain symbiotic relationships (Viprey et al., 1998), and that a given bacterium can engage in pathogenic and symbiotic interactions with different hosts (Preston, 2007). A symbiotic relationship also appears plausible given the known symbiotic associations of other verrucomicrobial species, such as Candidatus Xiphinematobacter with nematodes (Vandekerckhove et al., 2000), and the verrucomicrobial ectosymbiont that defends its ciliate host from predation (Petroni et al., 2000). In addition, a verrucomicrobial species occurs as a dominant member of the mucin-degrading bacterial population of the human intestine (Derrien et al., 2004). Future work includes determining the natural host range of $V$. spinosum, and the nature and route of the host interaction. This is a challenging goal given the many possible host taxa. However, our phylogenetic analysis of the V. spinosum T3SS proteins suggests that they are related to those found within Desulfovibrio and relatives, a group that includes known intestinal pathogens of vertebrates, as well as free-living strains. This suggests a number of possible avenues: to test vertebrate hosts for susceptibility to $V$. spinosum infection, and to test ingestion as an infection route in both vertebrate and invertebrate hosts. Interestingly, the role of FSHR-1 in pathogen defense in C. elegans is specific to the intestinal tract (Powell et al., 2009), consistent with V. spinosum acting through this tissue.

Another obvious goal is determination of the role of the predicted $V$. spinosum T3SS in the mortality observed in invertebrate infection models. The major hurdle to this work is that the development of genetic tools for $V$. spinosum is still in its infancy. Although a method for random transposon mutagenesis of the $V$. spinosum genome has been recently described (Domman et al., 2011), work is still underway to increase transformation efficiency so that mutant libraries of adequate size can be generated. In addition, neither transformation of $V$. spinosum cells with plasmid DNA nor targeted knock-out of specific genes via transposon mutagenesis has yet been achieved. With further progress in development of genetic tools for V. spinosum, it should also be possible to generate tagged $V$. spinosum mutants useful for fulfilling Koch's postulates (i.e., recovering the presumed pathogen/symbiont from the host) and determining whether the organism colonizes host tissue in the invertebrate infection models. In the absence of a selectable marker or fluorescent tag, it has proven difficult to quantify $V$. spinosum in worm or fly tissue due to overgrowth with normal fly/worm flora on artificial culture media. This question could also be pursued with FISH probing of host tissues with a $V$. spinosum-specific probe. An alternative, or complementary approach, to determining whether the putative $V$. spinosum T3SS is responsible for the observed invertebrate mortality would be to test for up-regulation of the relevant genes in the D. melanogaster or C. elegans infection models.

With the rapid development of sequencing technology, bacterial genome sequencing has revealed predicted pathogenesis/symbiosis determinants in several organisms not previously known to interact with eukaryotes (Pallen et al., 2005). Further examination of unpublished but publicly available genome sequences reveals the presence of such determinants in multiple other "free-living" genomes (http://img.jgi.doe.gov/cgi-bin/pub/ main.cgi). Here we map out a general methodology moving from bioinformatics to experimental analysis, taking advantage of established eukaryotic models from yeast to flies and worms to test the potential eukaryotic interactions of bacterial strains.

\section{MATERIALS AND METHODS BIOINFORMATIC PREDICTIONS AND PHYLOGENETIC ANALYSIS}

The V. spinosum genome sequence (accession ABIZ00000000) and the sequences of reference genomes were obtained from GenBank. Protein structure was predicted using the Phyre server (Kelley and Sternberg, 2009). Protein sequences of the T3SS ATPase (YscN) were identified using BLASTP (Altschul et al., 1990). The ATP synthase of E. coli associated with the flagellar apparatus was included in the analysis as an outgroup. Protein sequences were aligned using MUSCLE (Edgar, 2004) with default parameters. The WAG + I + GAMMA model (Whelan and Goldman, 2001) was determined as the model of best fit [according to AIC (Sugiura, 1978)] using the ProtTest tool (Abascal et al., 2005) with default parameters. Maximum likelihood phylogeny was obtained using PhyML software (Guindon and Gascuel, 2003) implementing the WAG + I + GAMMA evolutionary model, with 1000 bootstrap runs.

\section{BACTERIAL STRAINS AND CULTIVATION CONDITIONS}

Verrucomicrobium spinosum type strain DSM 4136 was grown in liquid M13 medium (Schlesner, 1987) incubated statically at $30^{\circ} \mathrm{C}$ for $72 \mathrm{~h}$.

\section{RNA ISOLATION AND RT-PCR}

Ten milliliters of $V$. spinosum culture was collected by centrifugation and frozen at $-80^{\circ} \mathrm{C}$ for $5 \mathrm{~min}$. Cells were thawed, $250 \mu \mathrm{l}$ TriZol reagent was added, and RNA isolation performed as recommended by the manufacturer. Pellets were re-suspended and incubated for $15 \mathrm{~min}$ at $55^{\circ} \mathrm{C}$ in $100 \mu \mathrm{l}$ RNase-free water, DNasetreated, and subsequently passed through an RNeasy mini kit (Qiagen) according to the manufacturer's instructions. Reverse 
transcription was performed using the iScript cDNA synthesis kit (Bio-rad) according to the manufacturer's instructions. PCR targeting VspC (ORF05910), VspQ (ORF05897), VspF1 (ORF05907), and VspF2 (ORF05908) was performed using V. spinosum cDNA or gDNA as the nucleic acid template. Reaction mixtures consisted of $1 \times$ PCR buffer (NEB), $1.5 \mathrm{mM} \mathrm{MgCl}_{2}, 10 \mu \mathrm{M}$ each primer (Table A3 in Appendix), $200 \mu \mathrm{M}$ each dNTP, $0.2 \mathrm{U}$ Taq DNA polymerase, $30 \mathrm{ng}$ of cDNA in a $50-\mu \mathrm{l}$ volume. Reaction conditions consisted of an initial denaturation step of $95^{\circ} \mathrm{C}$ for $3 \mathrm{~min}$ followed by 35 repeat cycles of $95^{\circ} \mathrm{C}$ for $10 \mathrm{~s}, 62^{\circ} \mathrm{C}$ for $10 \mathrm{~s}$, and $72^{\circ} \mathrm{C}$ for $20 \mathrm{~s}$, and a single final extension step at $72^{\circ} \mathrm{C}$ for $5 \mathrm{~min}$.

\section{YEAST TWO-HYBRID SCREENS}

Open reading frames were PCR-amplified from $V$. spinosum genomic DNA (gDNA). PCR was performed in a mixture of $1 \times$ iProof High-Fidelity DNA polymerase PCR reaction mix (Bio$\mathrm{Rad}), 25 \mu \mathrm{M}$ each primer, and approximately $30 \mathrm{ng}$ of $V$. spinosum gDNA in a final volume of $50 \mu \mathrm{l}$. Reaction conditions consisted of an initial denaturation step of $98^{\circ} \mathrm{C}$ for $30 \mathrm{~s}$ followed by 30 cycles of $98^{\circ} \mathrm{C}$ for $10 \mathrm{~s}, 54-60^{\circ} \mathrm{C}$ (see Table A3 in Appendix for primer sequences and annealing temperatures) for $10 \mathrm{~s}$ and $72^{\circ} \mathrm{C}$ for $1 \mathrm{~min}$, followed by a single final extension step of $72^{\circ} \mathrm{C}$ for $5 \mathrm{~min}$. PCR products were gel-purified using a QIAquick gel extraction kit (Qiagen), and cloned into pENTR/SD/D-TOPO (Invitrogen). E. coli NEB5 $\alpha$ chemically competent cells (NEB) were transformed with the resulting constructs to generate entry clones. Correct sequence and orientation of each ORF was confirmed by DNA sequencing of entry clones. ORF's were transferred to pDEST22 (Prey) and pDEST32 (Bait) vectors (Invitrogen) by LR recombination and specific protein-protein interactions were tested using the ProQuest Two-Hybrid System (Invitrogen), according to the manufacturer's instructions.

\section{YEAST T3SS EFFECTOR SCREEN}

Putative T3SS effectors were selected for yeast genetic screening (Lesser and Miller, 2001) if they showed high BLASTP similarity to known T3SS effectors in other organisms, or if they yielded high scores in criteria for T3SS effectors described previously (Schechter et al., 2006). PCR primers were designed to incorporate a yeast translation initiation sequence at the $5^{\prime}$ end of the ORF and the stop codon was removed to allow fusion with a V5 epitope and polyhistidine tag. Putative T3SS effectors (ORF01842, ORF04374, ORF05930) and controls (ORF03840, ORF04373, ORF05921) were amplified by PCR. PCR contained $1 \times$ ReadyMix PCR reaction mix (Sigma), $25 \mu \mathrm{M}$ each primer (Table A3 in Appendix), 30 ng V. spinosum gDNA, 3\% (v/v) DMSO, $1.5 \mathrm{mM} \mathrm{MgCl}_{2}$, and reaction conditions consisted of an initial denaturation step of $94^{\circ} \mathrm{C}$ for $5 \mathrm{~min}$, followed by 35 cycles of $94^{\circ} \mathrm{C}$ for $15 \mathrm{~s}, 56^{\circ} \mathrm{C}$ for $15 \mathrm{~s}$ and $72^{\circ} \mathrm{C}$ for $1 \mathrm{~min}$, and a single final extension step at $72^{\circ} \mathrm{C}$ for $5 \mathrm{~min}$. PCR products were gel-purified using a QIAquick gel extraction kit (Qiagen), cloned into pYES2.1 TOPO (Invitrogen). E. coli TOP10 chemically competent cells (Invitrogen) were transformed with the resulting constructs according to the manufacturer's instructions. Correct sequence and orientation of each ORF was confirmed by DNA sequencing. Plasmids were extracted from $5 \mathrm{ml}$ E. coli TOP10 using the QiaQuick plasmid miniprep kit (Qiagen). S. cerevisiae str. InvSc1 (Invitrogen) was transformed with approximately $1 \mu \mathrm{g}$ plasmid mixed with $100 \mu \mathrm{g}$ salmon sperm DNA, using a lithium acetate transformation protocol described by the manufacturer. Resulting transformants containing expression plasmids were selected by growth on SM-Ura media.

Saccharomyces cerevisiae InvSc1 clones containing expression plasmids were grown in $5 \mathrm{ml}$ non-inducing media (SMura + glucose) until culture had an $\mathrm{OD}_{600}$ of approximately 1.0. Cultures were normalized and inoculated into induction media (SM-ura + galactose) so that an $\mathrm{OD}_{600}$ of 0.05 was obtained. Tubes were incubated for $48 \mathrm{~h}$ at $30^{\circ} \mathrm{C}$ and the $\mathrm{OD}_{600}$ recorded. The final $\mathrm{OD}_{600}$ recorded was the average of two replicate cultures of 12 individual clones for each putative T3SS effector or control.

\section{FLY EXPERIMENTS}

Survival assays in D. melanogaster were performed as previously described (Schneider et al., 2007). 60-100 wild-type male Oregon $\mathrm{R}$ flies were assayed per condition. Flies were infected with either $V$. spinosum [concentrated in phosphate-buffered saline (PBS) to an $\mathrm{OD}_{600}$ of 1.0] or PBS alone and incubated at $29^{\circ} \mathrm{C}$. Death was recorded daily. Survival curves are plotted as Kaplan-Meier plots and statistical significance tested using log-rank analysis using GraphPad Prism software. All experiments were performed at least three times and yielded similar results.

\section{WORM EXPERIMENTS}

Strains were maintained according to standard procedures (Stiernagle, 2006) and cultured at $20^{\circ} \mathrm{C}$, with the exception of experiments conducted with CF512; such experiments were performed at $25^{\circ} \mathrm{C}$. Strains used or generated included the following: N2, wildtype; CF512, [fer-15(b26); fem-1(hc17)]; WY346, [fshr-1(ok778)]; WY335, [fshr-1(ok778); fdEx41] (this multi-copy extrachromosomal array contains the wild-type $f_{s h} r-1$ locus as well as a sur5::GFP marker; Cho et al., 2007). Bacterial killing assays were performed at $20^{\circ} \mathrm{C}$, unless otherwise noted, as previously described (Powell and Ausubel, 2007). V. spinosum was cultivated in liquid M13 medium as described above, while E. coli, S. aureus, and E. faecalis were cultivated in tryptic soy broth at $37^{\circ} \mathrm{C}$. After concentration of bacterial cultures by centrifugation, all bacterial strains were applied to NGM plates (Stiernagle, 2006) for the killing assays. Log-rank analysis was used to calculate mean survival for each population of worms, using the online server at http://bioinf.wehi.edu.au/software/russell/logrank/. Each population (biological replicate) was an average of two or three independent plates containing 50 worms each. Figures show data from representative biological replicates.

\section{ACKNOWLEDGMENTS}

This research was supported by the United States National Science Foundation (EPS-0447681). We would like to thank members of the Thorsness Lab in the Department of Molecular Biology, University of Wyoming, for assistance with experiments performed in yeast. 


\section{REFERENCES}

Abascal, F., Zardoya, R., and Posada, D. (2005). ProtTest: selection of best-fit models of protein evolution. Bioinformatics 21, 2104-2105.

Alberdi, M. P., Watson, E., McAllister, G. E., Harris, J. D., Paxton, E. A., Thomson, J. R., and Smith, D. G. (2009). Expression by Lawsonia intracellularis of type III secretion system components during infection. Vet. Microbiol. 139, 298-303.

Altschul, S. F., Gish, W., Miller, W., Myers, E. W., and Lipman, D. J. (1990). Basic local alignment search tool. J. Mol. Biol. 215, 403-410.

Brandt, S. M., Dionne, M. S., Khush, R. S., Pham, L. N., Vigdal, T. J., and Schneider, D. S. (2004). Secreted bacterial effectors and hostproduced Eiger/TNF drive death in a Salmonella-infected fruit fly. PLoS Biol. 2, e418. doi:10.1371/journal.pbio.0020418

Cho, S., Rogers, K. W., and Fay, D. S. (2007). The C. elegans glycopeptide hormone receptor ortholog, FSHR1 , regulates germline differentiation and survival. Curr. Biol. 17, 203-212.

Derrien, M., Vaughan, E. E., Plugge, C. M., and de Vos, W. M. (2004). Akkermansia muciniphila gen. nov., sp. nov., a human intestinal mucindegrading bacterium. Int. J. Syst. Evol. Microbiol. 54, 1469-1476.

Domman, D. B., Steven, B. T., and Ward, N. L. (2011). Random transposon mutagenesis of Verrucomicrobium spinosum DSM 4136(T). Arch. Microbiol. 193, 307-312.

Edgar, R. C. (2004). MUSCLE: multiple sequence alignment with high accuracy and high throughput. Nucleic Acids Res. 32, 1792-1797.

Galán, J. E., and Wolf-Watz, H. (2006). Protein delivery into eukaryotic cells by type III secretion machines. Nature 444, 567-573.

Gibson, G. R., Cummings, J. H., and Macfarlane, G. T. (1991). Growth and activities of sulphate-reducing bacteria in gut contents of healthy subjects and patients with ulcerative colitis. FEMS Microbiol. Lett. 86, 103-112.

Gibson, G. R., Cummings, J. H., Macfarlane, G. T., Allison, C., Segal, I., Vorster, H. H., and Walker, A. R. (1990). Alternative pathways for hydrogen disposal during fermentation in the human colon. Gut 31, 679-683.

Goldstein, E. J. C., Citron, D. M., Peraino, V. A., and Cross, S. A. (2003). Desulfovibrio desulfuricans bacteremia and review of human Desulfovibrio infections. J. Clin. Microbiol. 41, 2752-2754.
Guindon, S., and Gascuel, O. (2003). A simple, fast, and accurate algorithm to estimate large phylogenies by maximum likelihood. Syst. Biol. 52, 696-704.

Heidelberg, J. F., Seshadri, R., Haveman, S. A., Hemme, C. L., Paulsen, I. T., Kolonay, J. F., Eisen, J. A., Ward, N., Methe, B., Brinkac, L. M., Daugherty, S. C., Deboy, R. T., Dodson, R. J., Durkin, A. S., Madupu, R., Nelson, W. C., Sullivan, S. A., Fouts, D., Haft, D. H., Selengut, J., Peterson, J. D., Davidsen, T. M., Zafar, N., Zhou, L., Radune, D., Dimitrov, G., Hance, M., Tran, K., Khouri, H., Gill, J., Utterback, T. R., Feldblyum, T. V., Wall, J. D., Voordouw, G., and Fraser, C. M. (2004). The genome sequence of the anaerobic, sulfate-reducing bacterium Desulfovibrio vulgaris Hildenborough. Nat. Biotechnol. 22, 554-559.

Kelley, L. A., and Sternberg, M. J. E. (2009). Protein structure prediction on the web: a case study using the Phyre server. Nat. Protoc. 4, 363-371.

Kroll, J. J., Roof, M. B., Hoffman, L. J., Dickson, J. S., and Harris, D. L. (2005). Proliferative enteropathy: a global enteric disease of pigs caused by Lawsonia intracellularis. Anim. Health Res. Rev. 6, 173-197.

Lee, K. C., Webb, R. I., Janssen, P. H., Sangwan, P., Romeo, T., Staley, J. T., and Fuerst, J. A. (2009). Phylum Verrucomicrobia representatives share a compartmentalized cell plan with members of bacterial phylum Planctomycetes. BMC Microbiol. 9, 5. doi:10.1186/1471-2180-9-5

Lemaitre, B., Reichhart, J. M., and Hoffmann, J. A. (1997). Drosophila host defense: differential induction of antimicrobial peptide genes after infection by various classes of microorganisms. Proc. Natl. Acad. Sci. U.S.A. 94, 14614-14619.

Lesser, C. F., and Miller, S. I. (2001). Expression of microbial virulence proteins in Saccharomyces cerevisiae models mammalian infection. EMBO J. 20, 1840-1849.

Loubinoux, J., Bronowicki, J., Pereira, I. A. C., Mougenel, J., and Faou, A. E. (2002). Sulfate-reducing bacteria in human feces and their association with inflammatory bowel diseases. FEMS Microbiol. Ecol. 40, 107-112.

Martiny, J. B. H., and Field, D. (2005). Ecological perspectives on the sequenced genome collection. Ecol. Lett. 8, 1334-1345.

Pallen, M. J., Beatson, S. A., and Bailey, C. M. (2005). Bioinformatics, genomics and evolution of non-flagellar type-III secretion systems: a Darwinian perspective.
FEMS Microbiol. Rev. 29, 201-229.

Petroni, G., Spring, S., Schleifer, K. H., Verni, F., and Rosati, G. (2000). Defensive extrusive ectosymbionts of Euplotidium (Ciliophora) that contain microtubule-like structures are bacteria related to Verrucomicrobia. Proc. Natl. Acad. Sci. U.S.A. 97, 1813-1187.

Powell, J. R., and Ausubel, F. M. (2007). "Models of Caenorhabditis elegans infection by bacterial and fungal pathogens," in Innate Immunity, Methods in Molecular Biology, Vol. 415, eds J. Ewbank and E. Vivier (Totowa, NJ: Human Press), 403-427.

Powell, J. R., Kim, D. H., and Ausubel, F. M. (2009). The G protein-coupled receptor FSHR-1 is required for the Caenorhabiditis elegans innate immune response. Proc. Natl. Acad. Sci. U.S.A. 106, 2782-2787.

Preston, G. M. (2007). Metropoli$\tan$ microbes: type III secretion in multihost symbionts. Cell Host Microbe 2, 291-294.

Schechter, L. M., Vencato, M., Jordan, K. L., Schneider, S. E., Schneider, D. J., and Collmer, A. (2006). Multiple approaches to a complete inventory of Pseudomonas syringae pv. tomato DC3000 type III secretion system effector proteins. Mol. Plant Microbe Interact. 19, 1180-1192.

Schlesner, H. (1987). Verrucomicrobium spinosum gen. nov., sp. nov.: a fimbriated prosthecate bacterium. Syst. Appl. Microbiol. 10, 54-56.

Schneider, D. S., Ayres, J. S., Brandt, S. M., Costa, A., Dionne, M. S., Gordon, M. D., Mabery, E. M., Moule, M. G., Pham, L. N., and ShirasuHiza, M. M. (2007). Drosophila eiger mutants are sensitive to extracellular pathogens. PLoS Pathog. 3, e41. doi:10.1371/journal.ppat.0030041

Slagowski, N. L., Kramer, R. W., Morrison, M. F., LaBaer, J., and Lesser, C. F. (2008). A functional genomic yeast screen to identify pathogenic bacterial proteins. PLoS Pathog. 4, e9. doi:10.1371/journal.ppat.0040009

Stiernagle, T. (2006). "Maintenance of C. elegans," in WormBook (The C. elegans Research Community). 10.1895/wormbook.1.101.1, Available at: http://www.wormbook.org. [accessed February 11, 2006].

Sugiura, N. (1978). Further analysts of the data by akaike's information criterion and the finite corrections. Commun. Stat. Theory Methods 7, 13.

Troisfontaines, P., and Cornelis, G. R. (2005). Type III secretion: more systems than you think. Physiology (Bethesda) 20, 326-339.

Vandekerckhove, T. T., Willems, A., Gillis, M., and Coomans, A. (2000). Occurrence of novel verrucomicrobial species, endosymbiotic and associated with parthenogenesis in Xiphinema americanum-group species (Nematoda, Longidoridae). Int. J. Syst. Evol. Microbiol. 50, 2197-2205.

Viprey, V., Del Greco, A., Golinowski, W., Broughton, W. J., and Perret, X. (1998). Symbiotic implications of type III protein secretion machinery in Rhizobium. Mol. Microbiol.28, 1381-1389.

Whelan, S., and Goldman, N. (2001). A general empirical model of protein evolution derived from multiple protein families using a maximum-likelihood approach. Mol. Biol. Evol. 18, 691-699.

Willis, C. L., Cummings, J. H., Neale, G., and Gibson, G. R. (1997) Nutritional aspects of dissimilatory sulfate reduction in the human large intestine. Curr. Microbiol. 35, 294-298.

Zinkevich, V. V., and Beech, I. B. (2000). Screening of sulfate-reducing bacteria in colonoscopy samples from healthy and colitic human gut mucosa. FEMS Microbiol. Ecol. 34, 147-155.

Conflict of Interest Statement: The authors declare that the research was conducted in the absence of any commercial or financial relationships that could be construed as a potential conflict of interest.

Received: 21 June 2011; accepted: 30 September 2011; published online: 18 October 2011.

Citation: Sait M, Kamneva OK, Fay DS, Kirienko NV, Polek J, Shirasu-Hiza MM and Ward NL (2011) Genomic and experimental evidence suggests that Verrucomicrobium spinosum interacts with eukaryotes. Front. Microbio. 2:211. doi: 10.3389/fmicb.2011.00211

This article was submitted to Frontiers in Evolutionary and Genomic Microbiology, a specialty of Frontiers in Microbiology. Copyright (ㅇ 2011 Sait, Kamneva, Fay, Kirienko, Polek, Shirasu-Hiza andWard. This is an open-access article subject to a non-exclusive license between the authors and Frontiers Media SA, which permits use, distribution and reproduction in other forums, provided the original authors and source are credited and other Frontiers conditions are complied with. 


\section{APPENDIX}

Table A1 | Type III Secretion System structural genes and chaperones in the V. spinosum genome, predicted on the basis of primary sequence similarity (BLASTP comparison) and domain structure.

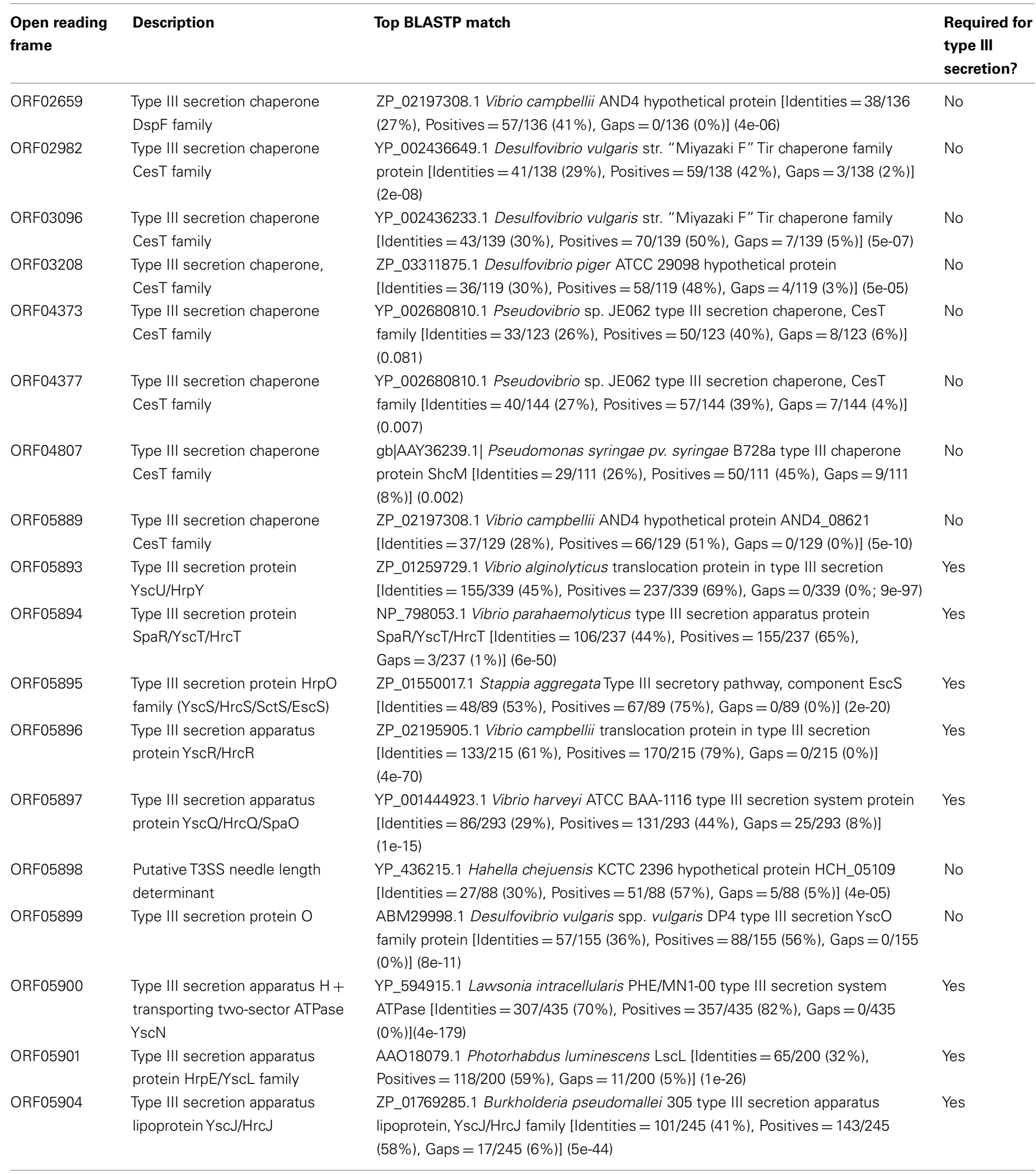


Table A1 | Continued

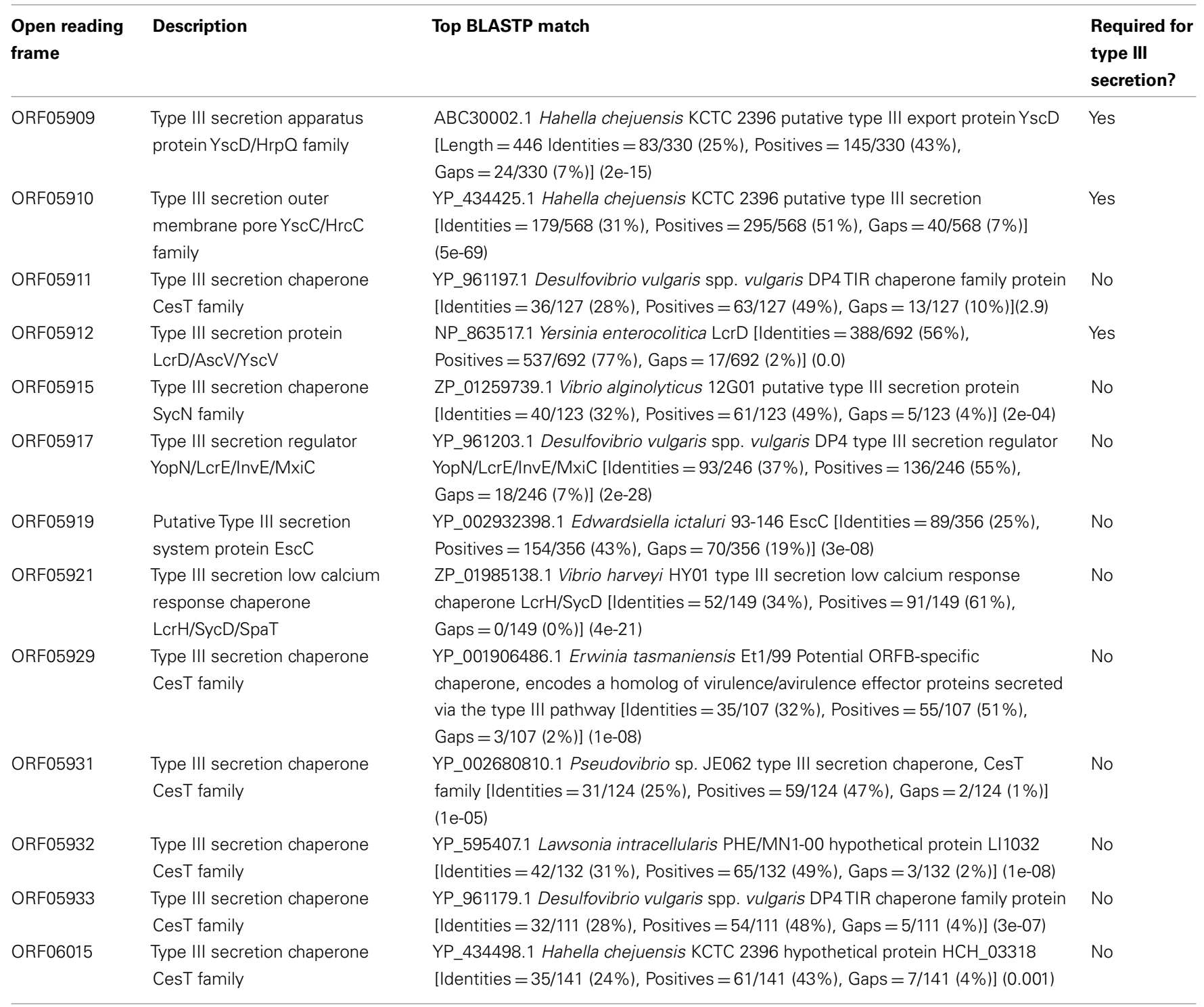


Table A2 | Results of yeast two-hybrid screening to detect protein:protein interactions between putative needle proteins and putative chaperones.

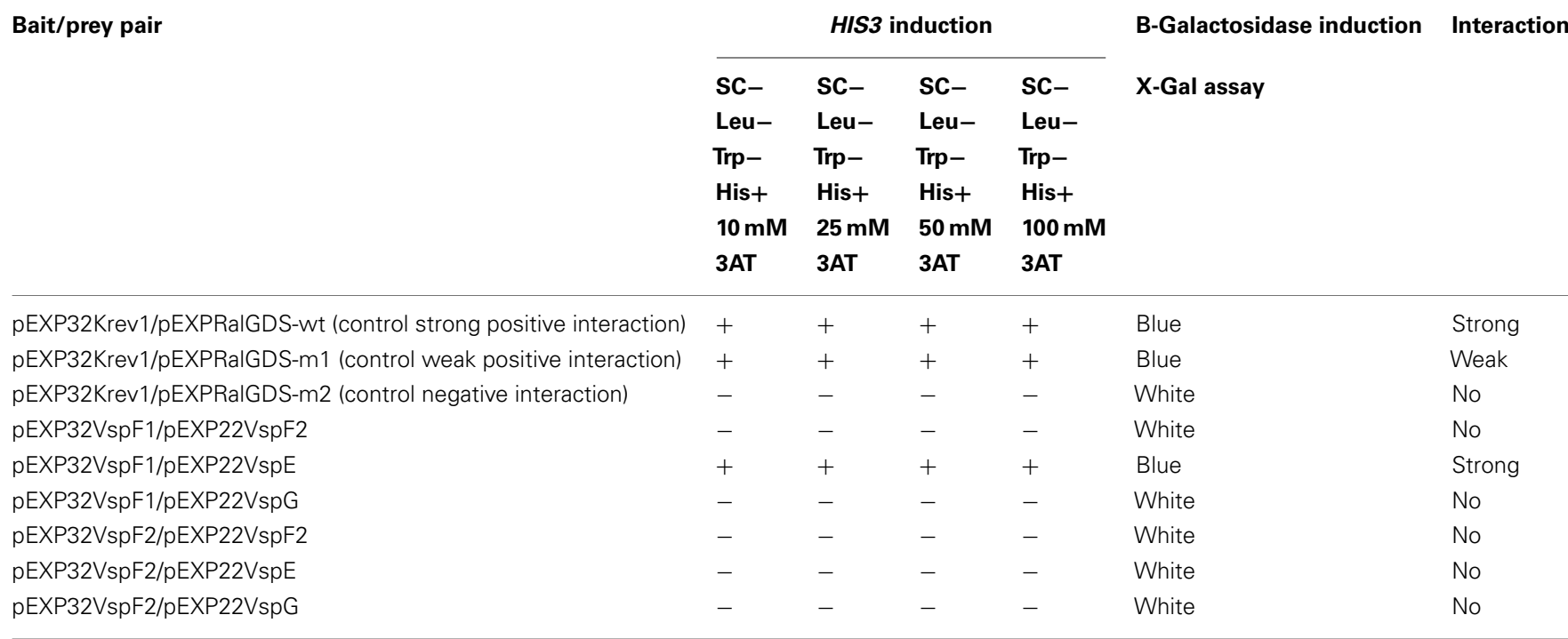

Table A3 | Oligonucleotide primers used in this study.

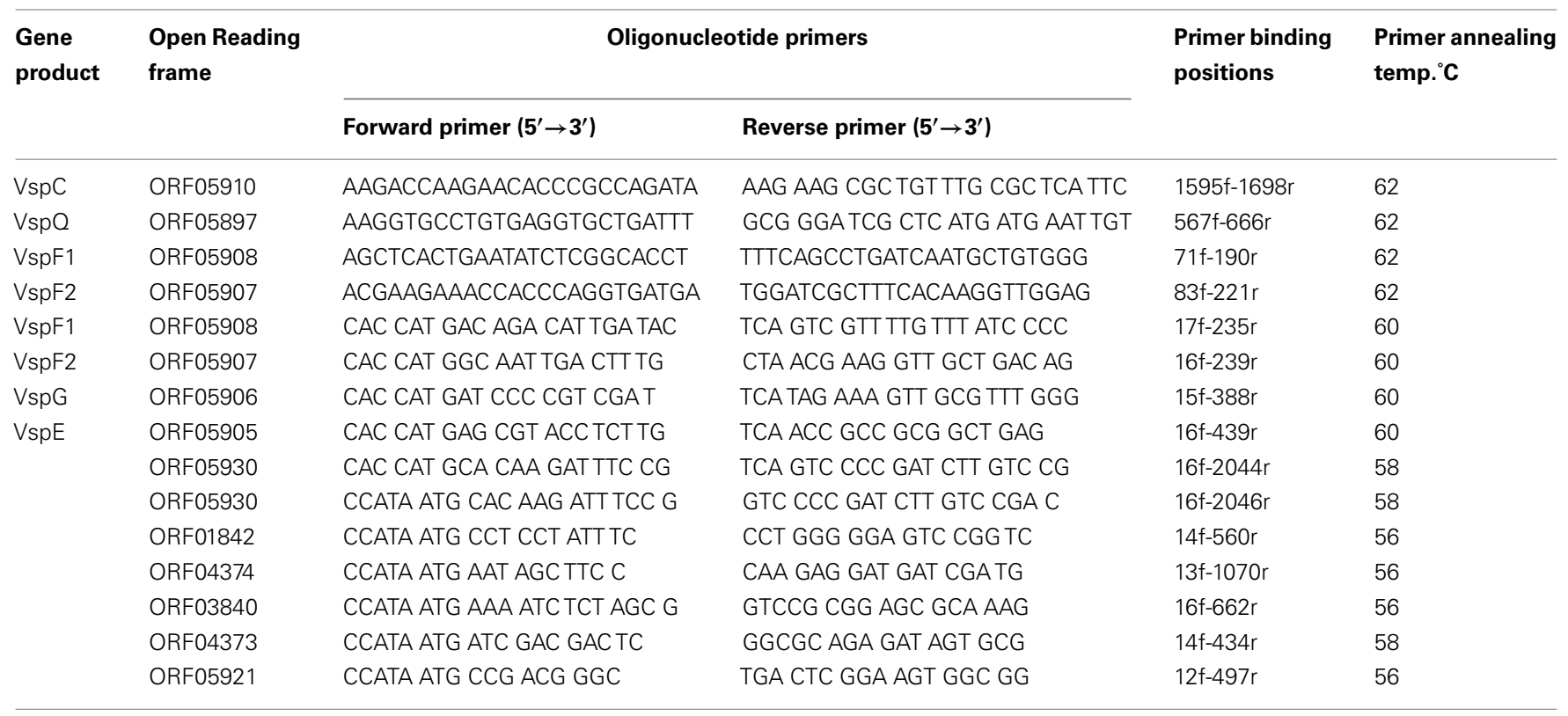



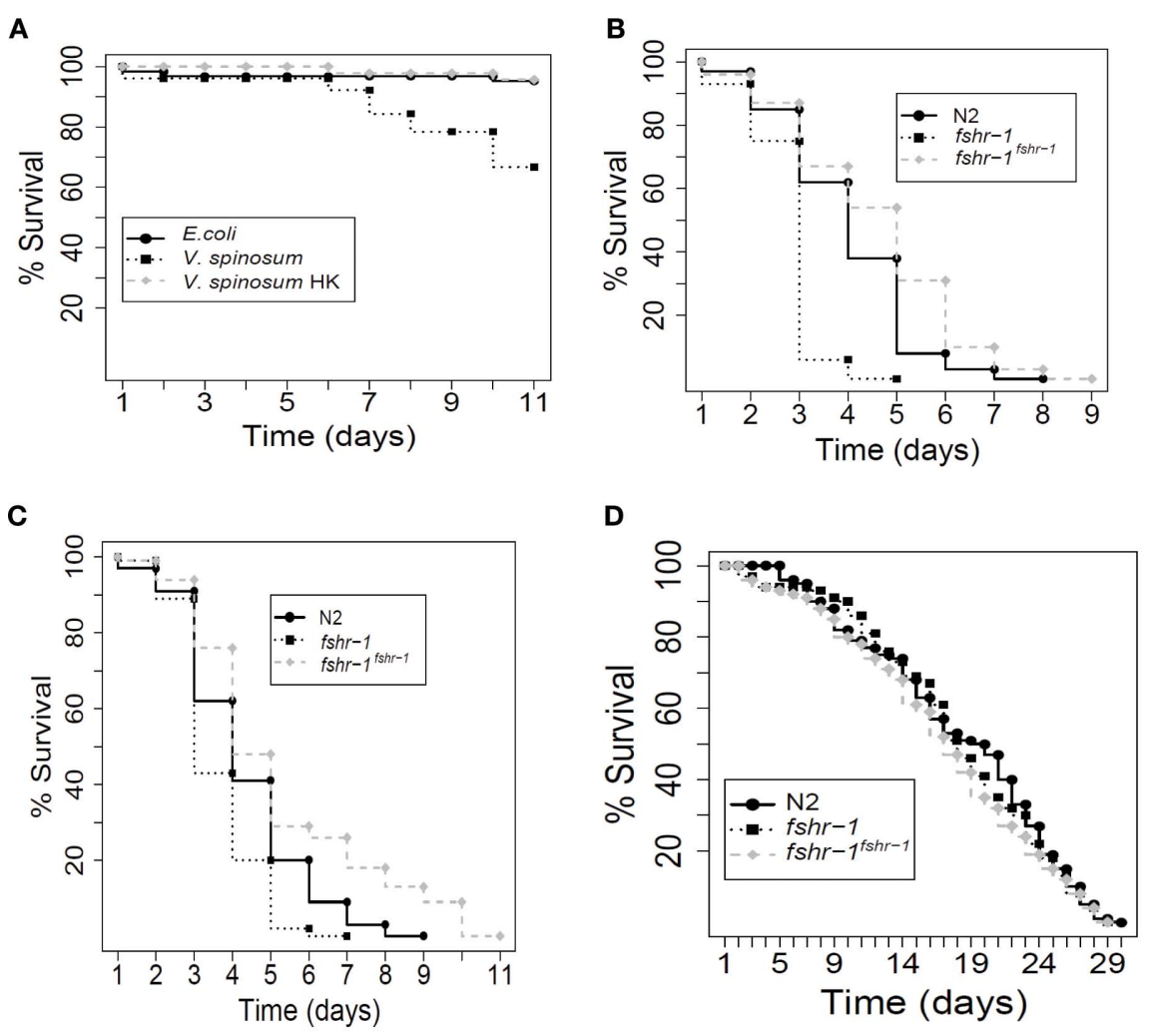

FIGURE A1 | Caenorhabditis elegans survival assays. Each experiment comprised approximately 100 worms. (A) Wild-type worms (N2) exposed to live $V$. spinosum died faster than worms exposed to $E$. coli, according to log-rank analysis $\left(p=3.71 \mathrm{e}^{-7}\right)$, whereas worms exposed to heat-killed $V$. spinosum died no faster than worms exposed to $E$. coli $(p=0.319)$. (B) When exposed to $S$. aureus, worm mutants hypersensitive to pathogens ( $\left.f_{s h}-1\right)$ died faster than N2 wild-type worms $\left(p=9.66 \mathrm{e}^{-15}\right)$. fshr-1 (ok778) worms over-expressing wild-type fshr-1 from a multi-copy transgenic array died more slowly than N2 wild-type

worms $(p=0.00114)$. (C) When exposed to $E$. faecalis, worm mutants hypersensitive to pathogens ( $f(s h r-1)$ died faster than $\mathrm{N} 2$ wild-type worms $\left(p=3.69 \mathrm{e}^{-5}\right)$. fshr-1 (ok778) worms over-expressing wild-type fshr-1 from a multi-copy transgenic array died more slowly than N2 wild-type worms $(p=0.000799)$. (D) When exposed to $E$. coli, there was no difference in the mortality rate between wild-type worms, fshr-1(ok778) mutants ( $p=0.541$, relative to wild-type), and fshr-1(ok778) mutants over-expressing wild-type fshr-1 from a transgenic multi-copy array ( $p=0.169$, relative to wild-type). 